\title{
Index of names
}

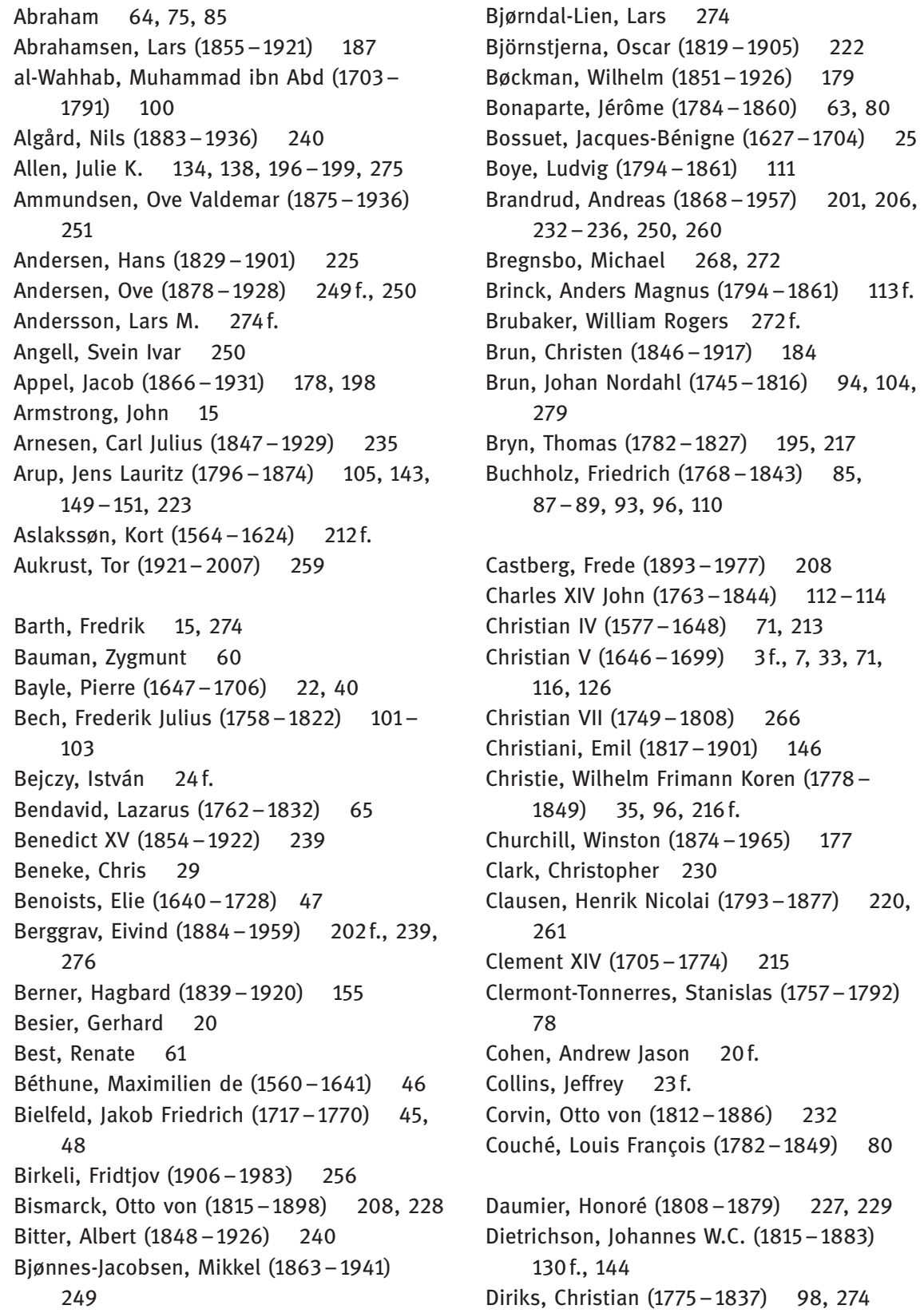

Ә OpenAccess. (C) 2021 Frode Ulvund, published by De Gruyter. (cc) BY-NC-ND This work is licensed under the Creative Commons Attribution-NonCommercial-NoDerivatives 4.0 License.

https://doi.org/10.1515/9783110657760-011 
Dittrich, Lisa 227

Djunkowski, Paul Maria Stefan (18211870) $223 \mathrm{f}$.

Dohm, Christian von (1751-1820) 56, 73, 119

Drake, Susanna 70

Dykes, George Parker (1814-1888) 127

Edmunds, George F. (1828-1919) 141

Elias, Norbert 25

Engelhardt, Juliane 269-272

Euchel, Gottleb (1767-1830) 73, 75, 91

Euchel, Isaac (1756-1804) 75

Fallize, Olaf (1844-1933) 226

Falsen, Christian Magnus (1782-1830) 55, 57, $92-97,218,273$

Farmer, Jared IX

Fawkes, Guy (1570-1606) 30

Feldbæk, Ole 265-267

Fichte, Johan Gottlieb (1762-1814) 51-54, $58,63-66,69,95,273$

Fjellbu, Arne (1890-1962) 255

Fleischer, Andreas (1878-1957) 201

Forsgren, John (1816-1890) 127

Forst, Rainer 22

Foss, Herman (1790-1853) 92

Frederick VI (1768-1839) 82-85, 113

Freece, Hans Peter (1879-1959) 175-178, 185, 198

Fries, Jakob (1773-1843) $94 \mathrm{f}$.

Frøvig, Daniel Andreas (1870-1954) 247

Fuchs, Eduard (1870-1840) IX

Fürst, Moses 72

Færden, Anders (1860-1939) 165, 167

Gillam, Bernhard (1856-1896) 132

Givens, Terryl 139

Gjesdahl, Johan Fredrik (1857-1944)

$178 \mathrm{f} ., 186,248$

Gleditsch, Jens Gran (1860-1931) 243, 247, 249

Glenthøj, Rasmus 265-268

Glogau, Heinrich Martin (1786-1821) 57, 92-98

Graetz, Friedrich (1840-1913) 132
Grattenauer, Karl Wilhelm Friedrich (17731838) 82

Gregory XVI (1765-1846) 219

Grell, Ole Peter 19

Gross, Michael B. 228

Haakon VII (1872-1957) 193

Habermas, Jürgen 21

Hafen, John (1856-1910) IX, 137

Hall, Stuart 16

Hallesby, Ole (1879-1961) 255, 260

Halling, Honoratius (1819-1886) 145

Hambro, Carl Joachim (1885-1964) 165, 251, 258-260

Hambro, Edvard (1782-1865) 98

Hambro, Nico (1861-1926) 165

Hammerich, Frederik (1809-1877) 148

Hansen, Mauritz (1794-1842) 225

Hansen, Peder (1746-1810) 100

Hansen, Peter O. (1818-1894) 127

Hanson, Hyrum Edwin 180

Harket, Håkon 55, 65, 92-94

Harvard, Jonas 275

Haslam, Gerald 150, 187

Hass, Ludvig Daniel (1808-1881) 149

Hauge, Hans Nielsen (1771-1824) 99-101

Hauge, Jens Kristian (1915-2006) 210

Hell, François (1731-1794) 64

Henriques, Jeremias 72,77

Henry IV (1553-1610) 19, 46

Hielm, Jonas Anton (1782-1848) 97

Hiorth, Albert (1876-1949) 237

Hoensbroch, Paul von (1852-1923) 232, 234

Hoffmann, Christhard 60f., 259, 274

Holberg, Ludvig (1684-1754) 41-44, 97

Horrebow, Otto (1769-1823) $88 \mathrm{f}$.

Hsia, Ronnie Po-Chia $26 \mathrm{f}$

Hudal, Aloysius (1885-1963) 259

Hutson, James $28 \mathrm{f}$.

Ihlen, Christian (1868-1958) 244

Indrebø, Ragnvald (1891-1984) 254

Ingerøe, Julie 156-159, 279

Jaabæk, Søren (1814-1894) 156

James II (1633-1701) 37 
Jansen-Fuhr, Jørgen (1878-1930) 174

Jensen, Peter Andreas (1812-1867) 153

Jensen, Thomas P. (1856-1952) 189

Jensenius, Caspar Holten (1821-1902)

$146,148,150$

Johnson, Gisle (1822-1894) 129

Joséphine of Leuchtenberg (1807-1876)

Kaartvedt, Alf 235

Kaas, Frederik Julius (1758-1827) $84 \mathrm{f}$, 101

Kaiser, Wolfram 230

Kamen, Henry 23

Kant, Immanuel (1724-1804) 68

Kaplan, Benjamin 25

Karp, Jonathan 50, 54

Kates, Gary $\quad 61,79$

Katz, Jacob 45 f., 48, 59, 68, 215

Katz, Per 71

Keppler, Joseph Ferdinand (1838-1894) 132

Kierkegaard, Søren (1813-1855) $209 \mathrm{f}$.

Klosterlasse (1538-1622) 211f., 232, 250

Knudsen, Christopher (1843-1915) $234-$ 236

Knudsen, Kai Birger (1903-1977) 208

Kolsrud, Oluf (1885-1945) 244, 247, $251 \mathrm{f}$.

Kortum, Ernst Traugott von (1742-1811)

$$
67-69,71,118
$$

Koselleck, Reinhardt 264, 276

Krünitz, Johann Georg (1728-1796) 62

Lammers, Gustav Adolph (1802-1878) 129

Larsen, Leroy (1887-1962) 174

Larson, Gustaf 180

Laurent, Johannes Th. (1804-1884) $219 \mathrm{f}$.

Laursen, John 25

Lehmann, Edvard (1862-1930) 247

Liljedahl, Edvard (1845-1924) 179

Liljefalk, Axel (1848-1915) 197

Linvald, Axel 82-85

Locke, John (1632-1704) 22, 35, 37-42, 44, 97

Lorenz, Chris $15 \mathrm{f}$.

Lortz, Joseph (1887-1975) 259

Louis XIV (1638-1715) 19, 47

Loyola, Ignatius (1491-1556) 157, 211, 224
Lumholtz, Nicolai (1729-1819) 103

Lunde, Johan (1866-1938) 243, 248

Lundegaard, Theis (1774-1856) $119 \mathrm{f}$.

Lützhøft, Hans (1857-1943) 198

Læstadius, Lars Levi (1800-1861) 130

Madison, James (1751-1836) $30 \mathrm{f}$.

Maroni, James (1873-1957) 202

Marshall, Thomas H. $271 \mathrm{f}$.

Martensen, Hans Lassen (1808-1884) 221

Mason, George (1725-1792) 30

Mauritzen, Anna Kristina 161

Meltzer, Fredrik (1779-1855) 117

Mendelssohn, Moses (1729-1786) 22, 72

Menzinger, Alois (1876-1941) 237

Meyer, Ludvig (1780-1854) 224

Meyer, Nathan Levin (c. 1756-1823) 72

Millot, Claude-François-Xavier (1726-1785) 215

Moe, Olaf (1876-1963) 244, 248, 255

Møller, H. O. Frimodt (1871-1934) 199,

Möllhausen, Balduin (1825-1905) $159 \mathrm{f}$.

Montz, Gottfried Ignatius (1813-1868) 223

Mortensen, Andreas (1849-1904) 156, 158 f., 279

Moses 58, 70, 76, 85-88, 93

Mowinckel, Sigmund (1884-1965) 239

Mulder, William $128 \mathrm{f}$.

Münter, Frederik (1761-1830) $82 \mathrm{f}$.

Muus, Rudolf (1862-1935) 171

Mynster, Jacob Peter (1775-1854) 108, 133f., 144, 219-221

Napoleon (1769-1821) 63, 78-83, 116, 279

Nast, Thomas (1840-1902) 231

Nederman, Cary 25

Nielsen, Lauritz (1538-1622) 211f., 232, 250

Nørregaard, Lauritz (1745-1804) 36, 267

Oftestad, Bernt $225 \mathrm{f}$.

Opper, Frederick Burr (1857-1937) 132

Ording, Hans (1884-1952) 255

Oscar I (1799-1859) 2

Ørsted, Anders Sandøe (1778-1860) 36, 108, 221 
Paine, Thomas (1737-1809) 21

Pan, Mallet du (1749-1800) 50

Pascal, Blaise (1623-1662) 213, 218

Paul 60, 179

Paul III (1468-1549) 211

Pavels, Claus (1769-1822) $96 \mathrm{f}$.

Petersen, Jacob Christian (1870-1964) 249

Pfannenstill, Magnus (1858-1940) 242

Pius XI (1857-1939) 247

Platou, Frederik (1811-1891) 149

Pontoppidan, Erik (1698-1764) 34

Porter, Roy $18 \mathrm{f} ., 26$

Pufendorf, Samuel (1632-1694) 35, 39-41, $43 \mathrm{f} ., 47,53,97$

Rawls, John 22

Reichenwald, Christian Birch (1814-1891) 150

Rein, Jonas (1760-1821) 92

Reinhart, Charles Stanley (1844-1896) 233

Reventlow, Eduard (1883-1963) 192, 194

Rhyn, Otto Henne am (1828-1914) $233 \mathrm{f}$.

Rian, Øystein 34

Richelieu, Armand Jean (1585-1642) 46

Riddervold, Hans (1795-1876) 143

Riesterer, Coelestin (1858-1938) $254 \mathrm{f}$.

Rönnow, Charles C. (1865-1945) 189

Roos, Heinrich (1904-1977) 208-210

Rosenius, Carl Olof (1816-1868) 130

Rossum, Wilhelm van (1854-1932) 241245, 247, 251

Rousseau, Jean-Jacques (1712-1778) 35, 270

Rouvroy, Louis de (1675-1755) 47

Rowlands, Richard (ca.1550-1640) 44

Rühs, Friedrich (1781-1820) 94

Rush, Benjamin (1746-1813) 32, 70

Said, Edward 62, 140

Samuelsen, Frederik (1865-1929) $197-$ 199

Saud, Abd al-Aziz ibn Muhammad ibn (?1803) 100

Saud, Muhammed ibn (?-1765) 100

Sauniers, Pierre-Yves 278

Schechter, Ronald 61,274
Scheer, Dominikus (1830-1907) 226

Schiller, Friedrich (1759-1805) 65

Schjelderup, Kristian (1894-1980) 255

Schlegel, Johan Frederik Wilhelm (17651836) 36,107

Schmidt-Phiseldek, Konrad (1770-1832) $85-87,97,105,118$

Schreiner, Karl (1865-1947) 175, 184

Schulz, Johann Heinrich (1739-1823) 64

Schweigaard, Christian (1838-1899) 234

Sehat, David 32

Seierstad, Andreas (1890-1975) 255

Seierstad, Ivar (1901-1987) 255

Seip, Karl (1850-1909) 183,

Seljaas, Helge 129

Sibbern, Valentin (1779-1853) 55, 217

Sieyès, Emmanuel-Joseph (1748-1836) $50-53,66,76,95,273$

Sigwardt, Mathias (1770-1840) 274

Simonsen, Kjetil Braut 275

Smith, Helmut Walser 228, 232

Smith, Joseph (1805-1844) 130 f, $134-$ 137, 140, 143, 148f, 206

Smoot, Reed (1862-1941) 160-162, 175, 189-195, $199 \mathrm{f}$.

Snow, Erastus (1818-1888) 127

Sparre, Ole Jacob (1831-1889) 155

Stabeck, Gustaf (1778-1831) 112

Stabell, Adolf Bredo (1807-1965) 224

Steenbuch, Henrik (1774-1839) 269

Steinsvik, Marta (1877-1950) 249, 253255

Stewart-Freece, Blanche (1878-1938) 175

Støylen, Bernt (1858-1937) 250

Stubbe, Henry (1632-1676) 38

Sundt, Eilert (1817-1875) 129, 150

Sutherland, George (1862-1942) 189

Svanberg, Ingvar 109

Sverdrup, Georg (1770-1850) 55, 103

Sverdrup, Jakob (1845-1899) $154 \mathrm{f}$.

Sybel, Heinrich Karl Ludolf von (18171895) 228

Söderblom, Nathan (1866-1931) 193, 241, 243

Sørensen, Jørgen Wurtz 127 
Sørensen, Søren Anton Wilhelm (17931853) 116,120

Sørflaten, Ole (1803-1889) 151

Tallien, Jean-Lambert (1767-1820) 273

Taranger, Absalon (1858-1930) 185, 244, 246

Thaarup, Thomas (1749-1821) 85, 87-88, 96,110

Thijs, Krijn 11

Thrane, Marcus (1817-1890) 13, 150

Tollefsen, Anders 231

Tollefsen, Anders (1849-1916) 231

Torp, Oscar (1893-1958) 208

Treschow, Niels (1751-1833) 103-105

Trygger, Ernst (1857-1943) 193

Tydén, Mattias 109

Ueland, Ole Gabriel (1799-1870) $119 \mathrm{f}$.

Ullmann, Viggo (1848-1910) 155, 226, 232, 236

Undset, Sigrid (1882-1949) 280

Valen-Sendstad, Olav (1904-1963) 256f., 259

Valentin, Hugo 110, 112, 114

Vatnaland, Elisæus (1892-1983) 260

Vechab, Abdul 100

Vig, Ole (1824-1857) 150
Vogt, Volrath (1817-1889) 153

Voltaire (1694-1778) 22f., 35

Wadensjö, Lars 238

Wallich, Wulf Lazarus (1756-1843) 73-77, 91

Walnum, Sven Borchmann Hersleb (18161889) 145

Weidemann, Lauritz (1775-1856) 55

Wergeland, Henrik (1808-1845) 115

Wergeland, Nicolai (1780-1848) 55

Werner, Yvonne Maria $\quad 221-222,261,275$

Widmer, Kurt 253

Widtsoe, John A. (1872-1952) 174, 191 195

Wikborg, Erling (1894-1992) 260

Wollmer, Lars (1879-1973) 242

Woodward, George Moutard (1760-1809) 70

Worm-Müller, Jacob S. 95

Wrangel, Herman (1857-1934) 190

Young, Brigham (1801-1877) 136f., 140, 145, 155, 157, 205

Zagorin, Perez 23

Åslev, P. E. $\quad$ 164, 170 f., 180 f., 186 